\title{
Autoantibodies in lung cancer: possibilities for early detection and subsequent cure
}

\author{
C J Chapman, ${ }^{1}$ A Murray, ${ }^{2}$ J E McElveen, ${ }^{2}$ U Sahin, ${ }^{3}$ U Luxemburger, ${ }^{3}$ Ö Türeci, ${ }^{3}$ \\ R Wiewrodt, ${ }^{3}$ A C Barnes, ${ }^{2}$ J F Robertson ${ }^{1,2}$
}

- A supplementary appendix is published online only at http:// thorax.bmi.com/content/vol63/ issue 3

${ }^{1}$ Division of Breast Surgery, The University of Nottingham, Nottingham, UK; ${ }^{2}$ Oncimmune Ltd, Nottingham City Hospital, Nottingham, UK; ${ }^{3}$ Department of Medicine III, Johannes Gutenberg University, Mainz, Germany

\section{Correspondence to:}

Dr C J Chapman, Division of Breast Surgery, Clinical Sciences Building, Nottingham City Hospital, Nottingham NG5 1PB, UK; caroline.chapman@ nottingham.ac.uk

Received 13 August 2007 Accepted 28 August 2007

Published Online First

11 October 2007

\section{ABSTRACT}

Background: People with lung cancer usually present at a late stage in the course of their disease when their chances of long-term survival are low. At present there is little to offer for early diagnosis, even in those at high risk of developing the disease. Autoantibodies have been shown to be present in the circulation of people with various forms of solid tumour before cancer-associated antigens can be detected, and these molecules can be measured up to 5 years before symptomatic disease.

Objective: To assess the potential of a panel of tumourassociated autoantibody profiles as an aid to other lung cancer screening modalities.

Methods: Plasma from normal controls $(n=50)$, patients with non-small cell lung cancer $(n=82)$ and patients with small cell lung cancer $(n=22)$ were investigated for the presence of autoantibodies to p53, cmyc, HER2, NY-ESO-1, CAGE, MUC1 and GBU4-5 by enzyme-linked immunosorbent assay.

Results: Raised levels of autoantibodies were seen to at least $1 / 7$ antigens in $76 \%$ of all the patients with lung cancer plasma tested, and $89 \%$ of node-negative patients, with a specificity of $92 \%$. There was no significant difference between the detection rates in the lung cancer subgroups, although more patients with squamous cell carcinomas (92\%) could be identified.

Conclusion: Measurement of an autoantibody response to one or more tumour-associated antigens in an optimised panel assay may provide a sensitive and specific blood test to aid the early detection of lung cancer.

Lung cancer is the largest cause of death from cancer world wide, killing around 900000 people every year. ${ }^{1}$ Tobacco smoking is estimated to cause around $90 \%$ of all cases in men and $80 \%$ in women ${ }^{2}$; however, other recognised risk factors for lung cancer include passive smoking; occupational exposures, especially to asbestos; and radon exposure. ${ }^{1}$ The latency period for lung cancers attributable to smoking is at least 20 years, ${ }^{1}$ yet lung cancer is often detected by a chest $x$ ray examination, by which time it is usually advanced and patients cannot be cured. Presently there is no accepted early diagnostic test, although screening trials using spiral CT in people "at risk" are continuing. ${ }^{4}$

Since the 1970s there has been little improvement in the 5-year survival rates for lung cancer, which are at their highest in the USA, but are still only $13 \%$ for men and $17 \%$ for women. ${ }^{5}$ In contrast, if lung cancer is diagnosed early, the 5year survival rate for UICC stages I and II is reported to be up to $50 \%$, and for stage 0 (when the cancer is found only in a local area and only in the first few layers of cells) approaches $80 \%$. There is therefore an urgent priority to produce a screening test which can identify patients with lung cancer in its early curable stage(s). ${ }^{5}$

An increasing number of reports describe the presence of a humoral immune response, in the form of autoantibodies, to tumour-associated antigens (TAAs) in lung and other solid tumours, ${ }^{6-8}$ and these autoantibodies have been described as being present before symptomatic disease. ${ }^{6-11}$ We have recently proposed the hypothesis that the measurement of such tumour-associated antibodies might be useful in breast cancer detection as a test to aid mammography, and have reported raised levels of autoantibodies to any one of a selected panel of TAAs in the circulation of patients with early invasive breast cancer, and ductal carcinoma in situ, taken at disease diagnosis. ${ }^{12}$ This report expands on these findings in lung cancer and describes a highly sensitive enzyme-linked immunosorbent assay (ELISA) which measures the presence of raised levels of autoantibodies to TAAs in the peripheral blood of patients with both small cell (SCLC) and non-small cell lung cancer (NSCLC).

The panel of seven antigens selected in this study comprises a number of well-recognised cancer-associated proteins, including the c-myc oncogene, whose expression is observed in a wide variety of tumours, and the p53 tumour suppressor gene, which is mutated in a large number of cancers. p53 was the first antigen described to elicit autoantibodies in cancer, ${ }^{13}$ and autoantibodies to p53 have also been shown to be raised in people who smoke both with and without evidence of cancer. ${ }^{14}{ }^{15}$ Such anti-p53 antibodies can, in some cases, be detected before the cancer diagnosis. ${ }^{11}{ }^{16}$

The panel also includes antigens which are overexpressed (HER2) or aberrantly expressed (MUC1) on the cell surface of many solid tumours, including lung, breast, stomach, prostate and ovary, ${ }^{17-19}$ and which have been shown to induce autoantibody responses in both breast $^{9}{ }^{12}$ and, in the case of MUC1, NSCLC. ${ }^{20}$ Also included are two previously described cancer testis antigens, NYESO-1, which has previously been shown to induce autoantibodies in NSCLC, ${ }^{21} 22$ and CAGE, which has been described as capable of inducing an autoantibody response in gastric, ${ }^{23}{ }^{24}$ pancreatic, ${ }^{25}$ and some lung cancers. ${ }^{21}{ }^{23}$ The final antigen in the panel is a recently identified protein GBU4-5, which was identified using SEREX technologies ${ }^{26}$ and described in a previous publication. ${ }^{24}$ GBU4-5 encodes a DEAD-box domain (like CAGE), but 
until 2003 had not been described as eliciting an autoantibody response. Autoantibody responses to different antigens that encode DEAD-box proteins have recently been described. ${ }^{22}{ }^{25}$ These DEAD-box-containing proteins are involved in RNA processing, ribosome assembly, spermatogenesis, embryogenesis and cell growth and division, ${ }^{27}$ and these proteins may well be important in the carcinogenic pathway. They seem to be immunogenic, cancer-specific and may provide diagnostic and potentially immunotherapeutic cancer targets.

\section{METHODS}

\section{Blood samples and patient details}

Blood samples were collected from 104 patients with lung cancer who were recruited after histopathological confirmation of the tumour. The study was approved by the institutional ethics committee of Rheinland-Pfalz. The smoking status of these patients was unknown. Samples (both before and after treatment) were taken at various time points after diagnosis (036 months). Normal blood plasma samples were also obtained from 50 healthy blood donors with no further clinical information; the age, sex and smoking status of these donors was unknown.

\section{Antigen production}

Specific cDNAs for p53, HER2 (extracellular domain), c-myc, NY-ESO-1, CAGE and GBU4-5 were subcloned, along with a small tag, into the PET21b expression vector (Novagen, Darmstadt, Germany). The recombinant proteins were expressed in BL21(DE3) bacteria (Novagen), grown in CYM media $^{28}$ and purified using His trap affinity columns (Amersham, Uppsala, Sweden) according to the manufacturer's protocols. A negative control protein was also produced (small tag alone) and purified under identical conditions. Proteins were refolded according to the manufacturer's protocol and tested for purity by sodium dodecyl sulphate-polyacrylamide gel electrophoresis (silver and Coomassie stained ${ }^{28}$ ) as well as by western blotting ${ }^{14}$ with appropriate mouse monoclonal antibodies. Only proteins that were $>95 \%$ pure were used in the assays. In this study assessment of MUC1 autoantibodies was made with an MUC1 "VNTR" 29 peptide (Peptide Protein Research Ltd, Fareham, UK) conjugated to bovine serum albumin (BSA), with BSA alone acting as a negative control.

\section{Autoantibody detection}

Autoantibody detection was by ELISA using microtitre plates coated with recombinant antigen or peptide according to inhouse protocols (patent pending). Remaining binding sites were blocked with high-salt buffer (HSB: phosphate-buffered saline (PBS) $+0.5 \mathrm{M} \mathrm{NaCl}, 0.2 \% \mathrm{w} / \mathrm{v}$ casein, $0.05 \%$ Tween 20 ). For all assays, freshly thawed plasma samples (diluted 1/100 in HSB) were incubated in triplicate at $50 \mu \mathrm{l}$ per well for 90 minutes, as well as appropriate control mouse monoclonal antibodies specific for capture proteins. Horseradish peroxidase (HRP)-

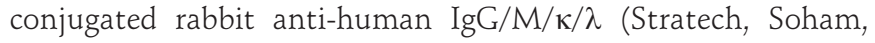
UK) or anti-mouse Ig antibodies (Dako, Ely, UK) were used as secondary antibodies at the dilution recommended by the manufacturer. Ready-prepared 3,3',5,5'-tetramethylbenzidine (Chemicon, Chandlers Ford, UK) was used as the chromogenic substrate for HRP, and absorbance values were determined after a 10-minute period at $\mathrm{A}_{650 \mathrm{~nm}}$. All incubations were carried out with shaking at room temperature and plates were washed three times with PBS containing Tween $20(0.1 \%$ v/v; Sigma, Poole, UK) between each step.

\section{Statistics}

Standard descriptive statistics-frequencies, mean and SDwere calculated to describe the study population. All analyses were performed using Microsoft Excel or Graph Pad software and were regarded as explorative. The number and proportion of positive samples were presented with the $95 \%$ exact confidence interval $(95 \% \mathrm{CI})$ for binomial proportions. ${ }^{30} \chi^{2}$ Tests were used to flag where the proportion of positive results was significantly different between cancer groups and the normal controls. For the panel 1 of 7 , testing was also performed to look at differences in positivity between lung cancer subgroups. This analysis was repeated on two subsets of data which consisted of pretreatment samples taken up to 1 and 6 months after diagnosis.

\section{Autoantibody assays}

Positive seroreactivity was defined as an optical density value greater than either the mean plus $2 \mathrm{SD}$ or mean plus $3 \mathrm{SD}$ of the normal cohort. The more stringent cut-off point (3SD) was applied to the c-myc, NY-ESO-1, p53, HER2 and MUC1 autoantibody assays, incorporating, on average, $99 \%$ of the distribution of the data. A cut-off value of an OD value greater than the mean plus 2SD of the normal population was applied to the autoantibody assays using the SEREX-identified antigen GBU4-5 and CAGE. ${ }^{24}$ In all autoantibody assays on normal plasma, values that were greater than the mean plus $5 \mathrm{SD}$ were removed to produce cut-off values, but were included in the analysis of specificity.

All assays were performed in triplicate on between two and four separate occasions. In addition, all positive plasma were confirmed with repeat testing, as were some negative plasma. Samples were designated positive for each separate autoantibody assay if there was a reproducible signal above the cut-off level of the normal group. For example, in patients with lung cancer a sample was only deemed reproducibly positive for an autoantibody assay if at least two out of two or three out of four interassay runs showed an increased value above the cutoff point. Where it was deemed that the result from one of the first runs was technically unassessable and where only two out of the remaining three assessable runs were increased, a fifth run was performed.

\section{RESULTS}

Table 1 shows patient details and tumour characteristics for both the total sample set $(n=104)$ and for those samples taken at, or close to, diagnosis $(n=70)$. No further details on the normal samples were available.

Table 2 shows levels of detection of autoantibodies against individual antigens in the two lung cancer disease groups and normal blood donor controls. The percentage of positive results in individual autoantibody assays ranged between 5\% (NYESO-1) and 36\% (MUC1) in the SCLC group and between 10\% (p53) and 34\% (MUC1) in the NSCLC group compared with between 0\% (GBU4-5, c-myc and MUC1) and 4\% (CAGE) in the normal controls. The highest level of autoantibody sensitivity was seen to the MUC1 peptide antigen for both forms of lung cancer. Specificity of the assay was calculated as the percentage of true negative samples that correctly gave a negative result. Individual assay specificity for each antigen varied from $96 \%$ to $100 \%$.

The patients with lung cancer (SCLC and NSCLC subgroups) differed significantly from the normal controls for all autoantibody assays $(p<0.05)$ apart from p53 in NSCLC and NY-ESO-1 
Table 1 Patient details and clinical characteristics of lung cancer samples

\begin{tabular}{|c|c|c|}
\hline Samples & NSCLC & SCLC \\
\hline \multicolumn{3}{|l|}{ All samples $(n=104)$} \\
\hline Total number (\%) & $82(100)$ & $22(100)$ \\
\hline Squamous cell carcinoma, n (\%) & $25(31)$ & NA \\
\hline Adenocarcinoma, n (\%) & $35(43)$ & NA \\
\hline Mixed (squamous+adeno), n (\%) & $1(1)$ & NA \\
\hline Large cell carcinoma, $\mathrm{n}(\%)$ & $1(1)$ & NA \\
\hline NOS, n (\%) & $20(24)$ & NA \\
\hline Age (years), mean (SD) & $64(10)$ & $62(11)$ \\
\hline Age range (years) & $36-83$ & $44-84$ \\
\hline Male, n (\%) & $57(70)$ & $14(64)$ \\
\hline LN negative, $\mathrm{n}(\%)$ & $12(15)$ & $0(0)$ \\
\hline \multicolumn{3}{|l|}{ Defined samples ${ }^{*}(n=70)$} \\
\hline Total number (\%) & $55(100)$ & $15(100)$ \\
\hline Squamous cell carcinoma, n (\%) & $16(29)$ & NA \\
\hline Adenocarcinoma, n (\%) & $24(44)$ & NA \\
\hline NOS & $15(27)$ & NA \\
\hline \multicolumn{3}{|l|}{ Stages, n (\%) } \\
\hline 1 & $5(9)$ & 0 \\
\hline$\|$ & $4(7)$ & 0 \\
\hline III & $13(24)$ & $3(20)$ \\
\hline IV & $27(49)$ & $11(73)$ \\
\hline Unknown & $6(11)$ & $1(7)$ \\
\hline Age (years), mean (SD) & $63(11)$ & $61(10)$ \\
\hline Age range (years) & $36-83$ & $52-75$ \\
\hline Male, n (\%) & $38(70)$ & $10(67)$ \\
\hline Pretreatment samples, n (\%) & $34(62)$ & $13(87)$ \\
\hline
\end{tabular}

${ }^{*}$ Samples taken within 4 (SCLC) or 6 (NSCLC) months of diagnosis date. LN, lymph node; NOS, not otherwise specified; NSCLC, non-small cell lung cancer; SCLC, small cell lung cancer.

in the case of SCLC. Only four of the normal cohort (8\%) had raised levels of autoantibodies to any one of the TAAs and one of these also had autoantibodies to multiple TAAs (p53, c-myc, HER2 and CAGE) (fig 1), which is rarely seen in normal samples. ${ }^{12}$ No clinical data were available for these four subjects.

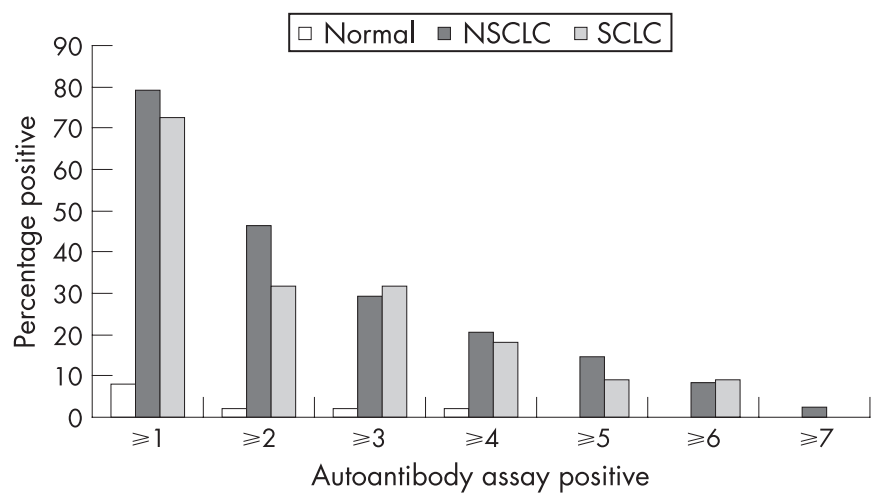

Figure 1 Autoantibody panel assay sensitivity. Percentage of each patient group positive for one or multiple autoantibodies. Positivity was defined as a value greater than the mean of the normal population plus 2SD for the SEREX-identified antigens CAGE and GBU4-5 or mean plus 3SD for the other assays. SCLC, small cell lung cancer; NSCLC, nonsmall cell lung cancer.

Combination of these seven autoantibody assays enhanced the autoantibody detection of cancer, with a panel sensitivity of $76 \%$ and panel specificity of $92 \%$. In most cases the levels of autoantibody responses to individual antigens in the cancer cases were significantly different from the normal control plasma $(p<0.05)$, but their importance in the panel assay varied. For example, raised levels of autoantibodies to the HER2 antigen were seen in $12 \%$ of the lung cancers; however, if the HER2 antigen was removed from the panel the detection rate and overall specificity for the NSCLC samples remained the same, and the overall sensitivity of the panel for all lung cancers only dropped from $76 \%$ to $74 \%$. This was reflected by the observation that a restricted panel assay consisting of the four antigens CAGE, GBU4-5, NY-ESO-1 and MUC1 exhibited only a slightly reduced sensitivity of $68 \%$ with an increased specificity of $94 \%$ (table 2 ).

In almost half of the seropositive individuals in panel 1 of 7 , autoantibodies were raised to a second antigen with similar

Table 2 Frequency of autoantibodies to tumour-associated antigens

\begin{tabular}{|c|c|c|c|c|c|c|c|c|c|c|}
\hline \multirow[b]{2}{*}{ Group } & \multirow[b]{2}{*}{ Total } & \multicolumn{9}{|c|}{ Number and percentage positive (with $95 \%$ exact confidence interval) } \\
\hline & & CAGE & GBU 4-5 & HER2 & p53 & c-myc & NY-ESO-1 & MUC1 & Panel 1/4 & Panel 1/7 \\
\hline \multicolumn{11}{|l|}{ All LC } \\
\hline $\mathrm{n}$ & 104 & $29 * * *$ & $21^{* * *}$ & $13^{* *}$ & $12^{*}$ & $12^{* * *}$ & $19 * * *$ & $36 * * *$ & $71^{* * *}$ & $79 * * *$ \\
\hline$\%+$ ve $(95 \% \mathrm{Cl})$ & & $28(20$ to 38$)$ & 20 (13 to 29$)$ & $13(7$ to 20$)$ & $12(6$ to 19$)$ & $12(6$ to 19$)$ & 18 (11 to 27$)$ & 35 (26 to 45$)$ & 68 (58 to 77$)$ & 76 (67 to 84 ) \\
\hline \multicolumn{11}{|l|}{ SCLC } \\
\hline$n$ & 22 & $5^{*}$ & $4^{* *}$ & $4^{*}$ & $4^{*}$ & $2^{*}$ & $1 \mathrm{NS}$ & $8^{* * *}$ & $12^{* * *}$ & $15^{* * *}$ \\
\hline$\%+$ ve $(95 \% \mathrm{Cl})$ & & 23 (8 to 45 ) & 18 (5 to 40$)$ & 18 (5 to 40$)$ & 18 (5 to 40$)$ & 9 (1 to 29$)$ & $5(0$ to 23$)$ & 36 (17 to 59$)$ & 55 (32 to 76$)$ & 68 (45 to 86$)$ \\
\hline \multicolumn{11}{|l|}{ NSCLC } \\
\hline $\mathrm{n}$ & 82 & $24^{* * *}$ & $17^{* * *}$ & $9 *$ & $8 \mathrm{NS}$ & $10^{* * *}$ & $18^{* * *}$ & $28^{* * *}$ & $59 * * *$ & $64^{* * *}$ \\
\hline$\%$ +ve $(95 \% \mathrm{Cl})$ & & $29(20$ to 40$)$ & 21 (13 to 31$)$ & 11 (5 to 20$)$ & 10 (4 to 18 ) & 12 (6 to 21$)$ & 22 (14 to 32$)$ & 34 (24 to 45$)$ & 72 (61 to 81$)$ & 78 (68 to 86$)$ \\
\hline \multicolumn{11}{|l|}{ Normal } \\
\hline $\mathrm{n}$ & 50 & 2 & 0 & 1 & 1 & 0 & 1 & 0 & 3 & 4 \\
\hline$\%+$ ve $(95 \% \mathrm{Cl})$ & & $4(0$ to 14$)$ & $0(0$ to 7$)$ & $2(0$ to 11$)$ & $2(0$ to 11$)$ & $0(0$ to 7$)$ & $2(0$ to 11$)$ & $0(0$ to 7$)$ & $6(1$ to 17$)$ & $8(2$ to 19$)$ \\
\hline Specificity (\%) & 50 & 96 & 100 & 98 & 98 & 100 & 98 & 100 & 94 & 92 \\
\hline
\end{tabular}

Number ( $\mathrm{n}$ ) and percentage positivity (\% +ve) with $95 \%$ confidence interval $(95 \% \mathrm{Cl})$ in each patient group. Positivity was defined as a value greater than the mean of the normal population plus $2 \mathrm{SD}$ for GBU4-5 and CAGE, or mean plus $3 \mathrm{SD}$ for the other assays.

${ }^{*}$ Denotes $p$ value relative to normal control plasma. ${ }^{*} \mathrm{p}<0.05$; ${ }^{*} \mathrm{p}<0.01 ;{ }^{* *} \mathrm{p}<0.001$; NS, not significant $\mathrm{p}>0.05\left(\chi^{2}\right.$ analysis).

All LC, all lung cancer samples tested; SCLC, small cell lung cancer; NSCLC, non-small cell lung cancer; Normal, normal blood donor plasma; Panel 1/4 or 1/7 denotes raised levels of at least one autoantibody of the panel; panel of 4: CAGE, GBU4-5; NY-ESO-1, MUC1 antigens only. 
Table 3 Autoantibody panel sensitivity by lung cancer subgroup

\begin{tabular}{|c|c|c|c|}
\hline \multirow[b]{3}{*}{ Group } & \multicolumn{3}{|c|}{ Autoantibody positivity population (panel of 7) } \\
\hline & \multirow{2}{*}{$\begin{array}{l}\text { All samples } \\
0-36 \text { months }\end{array}$} & \multirow{2}{*}{$\begin{array}{l}\text { Subset 0-1 } \\
0-1 \text { month } \\
\text { Pre-Tx }\end{array}$} & \multirow{2}{*}{$\begin{array}{l}\text { Subset 0-6 } \\
0-6 \text { months } \\
\text { Pre-Tx }\end{array}$} \\
\hline & & & \\
\hline \multicolumn{4}{|l|}{ SCLC } \\
\hline Number +ve & $15 / 22$ & $5 / 8$ & $9 / 13$ \\
\hline$\%+$ ve $(95 \% \mathrm{Cl})$ & 68 (45 to 86$)$ & 63 (24 to 91$)$ & 69 (39 to 91$)$ \\
\hline \multicolumn{4}{|l|}{ NSCLC } \\
\hline \multicolumn{4}{|l|}{ Squamous } \\
\hline Number +ve & $23 / 25$ & $5 / 5$ & $11 / 12$ \\
\hline$\%+$ ve $(95 \% \mathrm{Cl})$ & $92(74$ to 99$)$ & 100 (48 to 100$)$ & 92 (62 to 100$)$ \\
\hline \multicolumn{4}{|l|}{ Adeno } \\
\hline Number +ve & $27 / 35$ & $5 / 7$ & $13 / 16$ \\
\hline$\%+$ ve $(95 \% \mathrm{Cl})$ & 77 (60 to 90$)$ & 71 (29 to 96$)$ & 81 (54 to 96$)$ \\
\hline \multicolumn{4}{|l|}{ NOS } \\
\hline Number +ve & $14 / 22$ & $0 / 2$ & $2 / 6$ \\
\hline$\%+$ ve $(95 \% \mathrm{Cl})$ & 64 (41 to 83 ) & 0 (0 to 84$)$ & 33 (4 to 78 ) \\
\hline \multicolumn{4}{|l|}{ All $L C$} \\
\hline Number +ve & $79 / 104$ & $15 / 22$ & $35 / 47$ \\
\hline$\%+v e(95 \% \mathrm{Cl})$ & 76 (67 to 84 ) & 68 (45 to 86$)$ & $74(60$ to 86$)$ \\
\hline $\mathrm{p}$ Value from $\chi^{2}$ test $^{*}$ & 0.107 & 0.211 & 0.285 \\
\hline
\end{tabular}

Number and percentage positivity (\% +ve) with $95 \%$ confidence interval $(95 \% \mathrm{Cl})$ in each patient group using panel 1/7. Analysis repeated on two subset sample populations taking only pretreatment (pre-Tx) samples up to 1 month and 6 months from diagnosis.

${ }^{*} \chi^{2}$ Test across cancer subgroups noting that NOS was pooled with adeno in the subset analyses owing to small numbers.

All LC, all lung cancer samples tested; SCLC, small cell lung cancer; NSCLC, non-small cell lung cancer; Adeno, adenocarcinoma; Squamous, squamous cell carcinoma; NOS, not otherwise specified.

patterns in both NSCLC and SCLC samples, while only one of the 50 normal plasma samples exhibited more than one autoantibody response (fig 1).

The sensitivity of $76 \%$ (95\% CI $67 \%$ to $84 \%$ ) that was achieved by panel 1/7 in all 104 lung cancer samples was similar for the subsets of data where samples were taken before treatment, with sensitivity estimated at 68\% (95\% CI $45 \%$ to $86 \%$ ) and $74 \%(95 \%$ CI $60 \%$ to $86 \%$ ) for samples within 1 and 6 months of diagnosis, respectively (table 3). For all samples, sensitivity ranged from $64 \%$
(95\% CI $41 \%, 83 \%)$ for the NSCLC NOS group to $92 \%(95 \% \mathrm{CI}$ $74 \%$ to $99 \%$ ) for the NSCLC squamous group. Similar patterns were seen in the subsets. However, the differences in sensitivity between cancer subgroups was only borderline significant ( $p=0.107$ for all samples). There was also no apparent difference in detection rates if the lung cancer samples were subdivided by patient age or sex (data available in web appendix at http:// thorax.bmj.com/supplemental).

95\% Confidence intervals showed that even when sample numbers were small-for example, in pretreatment squamous cell carcinoma samples taken within 6 months of diagnosis (sensitivity $92 \%, \mathrm{n}=12$ ), it would be expected that over $62 \%$ of cases would have raised levels of autoantibodies using this panel of seven antigens (95\% CI $62 \%$ to $100 \%$ ). When all the squamous cell carcinoma samples were analysed this $92 \%$ detection rate was maintained, but with increased confidence in the result (23/25 autoantibody positive samples, $95 \%$ CI $74 \%$ to 99\%) (table 3).

The levels of sensitivity appeared to be maintained regardless of the lung cancer stage, though further confirmatory data is required (table 4). All three stage I and all three stage II pretreatment NSCLCs were detectable, giving a sensitivity estimated at $100 \%$, but the wide confidence interval $(95 \%$ CI $29 \%$ to $100 \%$ ) reflects the limited samples and need for further data. Eight out of nine lymph node negative pretreatment NSCLC samples also had raised levels of autoantibodies, leading to an estimated sensitivity of $89 \%$ (95\% CI $52 \%$ to $100 \%$ ) (data not tabulated).

\section{DISCUSSION}

Autoantibodies were produced to all seven of the TAAs studied. The presence of raised levels of autoantibodies to the known TAAs-p53, c-myc, HER2, MUC1 and NY-ESO-1-were in broad agreement with published data for individual autoantibody assays in both breast (reviewed by Storr et a $\mathbf{l}^{8}$ ) and other cancers such as colorectal, gastric, prostate, liver and lung. ${ }^{71} 32$ It confirms our findings and those of others that although measurement of autoantibodies to a single TAA is possible, the low sensitivity renders single autoantibody measurements of little use for screening and early diagnosis of cancer. Combination of these autoantibodies, together with the SEREX-identifiable antigens CAGE and GBU4-5 into a panel assay test provides an excellent level of sensitivity for the detection of lung cancer. Analysis of autoantibodies to the full panel of seven antigens resulted in $75 \%$ of all lung cancer

Table 4 Autoantibody assay panel sensitivity by tumour stage

\begin{tabular}{|c|c|c|c|c|c|c|}
\hline \multirow[b]{2}{*}{ Group } & \multirow[b]{2}{*}{ Subgroup } & & \multicolumn{4}{|c|}{ Autoantibody positivity population (panel of 7) } \\
\hline & & & Stage I & Stage II & Stage III & Stage IV \\
\hline SCLC & & Number +ve & 0 & 0 & $2 / 3$ & $7 / 11$ \\
\hline \multirow[t]{2}{*}{ SCLC } & Pre-Tx & Number +ve & 0 & 0 & $1 / 1$ & $7 / 11$ \\
\hline & $\mathrm{n}=12$ & $\%+$ ve $(95 \% \mathrm{Cl})$ & & & $100(3$ to 100$)$ & 64 (31 to 89$)$ \\
\hline NSCLC & $\mathrm{n}=49$ & $\%+$ ve $(95 \% \mathrm{Cl})$ & 80 (28 to 99$)$ & $100(40$ to 100$)$ & 77 (46 to 95$)$ & 78 (58 to 91$)$ \\
\hline \multirow[t]{2}{*}{ NSCLC } & Pre-Tx & Number +ve & $3 / 3$ & $3 / 3$ & $5 / 7$ & $15 / 20$ \\
\hline & $\mathrm{n}=33$ & $\%$ +ve $(95 \%$ Cl) & $100(29$ to 100$)$ & 100 (29 to 100$)$ & 71 (29 to 96$)$ & 75 (51 to 91 ) \\
\hline
\end{tabular}

Number and percentage positivity (\% +ve) with $95 \%$ confidence interval $(95 \% \mathrm{Cl})$ in each tumour stage (where known) using panel 1/7. Stage taken at diagnosis date follows from T,N,M-staging. SCLC, small cell lung cancer; NSCLC, non-small cell lung cancer; $95 \% \mathrm{Cl}$ denotes the $95 \%$ confidence interval; Pre-Tx, pretreatment samples.

Samples taken within 6 months of diagnosis for NSCLC, and within 4 months of diagnosis for SCLC. 
samples being identified, and $92 \%$ of squamous cell carcinomas with a specificity for cancer of $92 \%$, making this a test with potential clinical impact.

A previous study by Zhang et al demonstrated autoantibody reactivity to a panel of seven cancer-associated antigens, which differed from the antigens investigated here in all but p53 and cmyc. Their reported levels of sensitivities and specificities in lung cancer were $68 \%$ and $89-90 \%$ (depending on the normal group), respectively, which are similar to those we have demonstrated. However, the study numbers were smaller than those used here and no data on the stage, grade or subtype of the cancers tested were available.

This level of sensitivity of the assay for the identification of lung cancer was maintained in the subset of samples taken at or within 1 month of their cancer diagnosis. As it is unlikely that these autoantibodies were generated at the time of clinical presentation, the implications are that the antibodies would be present before diagnosis. Published evidence suggests that autoantibodies can be detected a number of years before clinical symptoms are observed, ${ }^{911}$ and affirmation of the results presented here, in prediagnostic samples, would confirm the utility of such an autoantibody test in lung cancer.

Although almost all the autoantibody assays measured significant responses in the plasma of patients with cancer, the individual sensitivity of each assay within the panel varied. Measurement of p53, c-myc and HER2 did not add significantly to the panel assay and increased sensitivity may be possible through substitution of these more general cancer antigens by other lung cancer-specific antigens in a panel assay. In contrast, measurement of the autoantibody response to the MUC1 core peptide was integral to the panel assays and was the most sensitive assay in all the histological subtypes of the lung samples studied. Measurement of the autoantibody responses to the cancer testis antigens was also important. A previous study investigated the presence of autoantibodies to NY-ESO-1 antigen in NSCLCs and SCLCs and observed levels of sensitivity and specificity similar to those reported here, finding that patients with SCLC had antibodies to NY-ESO-1 less frequently than patients NSCLC. ${ }^{19}$ In NSCLC the expression of NY-ESO-1 has been reported to be an independent prognostic marker of worse outcome in cases with adenocarcinoma, ${ }^{33}$ clinical followup data were not available on the population of patients reported in this study to confirm this previous finding.

Autoantibody responses to the DEAD-box proteins CAGE and the new cDNA GBU4-5 were also highly sensitive and specific in this study. The DEAD-box cancer testis antigen CAGE (DDX58) has previously been shown to be expressed in a number of cancers, including gastric, cervical and lung cancer tissue and cell lines, and autoantibodies have been reported to this protein in some but not all of the cancers samples studied, ${ }^{21}$ whereas autoantibodies to GBU4-5 have not been investigated in other cancers. We have preliminary data on the presence of autoantibodies to CAGE and GBU4-5 in patients with primary invasive breast cancer ( $\mathrm{n}=51$, full manuscript in preparation). These data did not show raised levels of autoantibodies to these antigens when compared with age-matched normal sera $(n=31) \quad(C A G E$ : sensitivity $6 \%$, specificity 97\%; GBU4-5: sensitivity $4 \%$, specificity $94 \%$ ). These observations suggest that distinguishing different solid tumour types may be aided by measuring autoantibodies to different DEAD-box-encoding proteins within a panel assay, although more data would be needed to confirm this.

Other antigens such as p53 and c-myc are more general cancer antigens and autoantibody responses to p53 have been reported to be raised in some people who smoke. ${ }^{14}$ At this time it is difficult to be certain whether p53 autoantibodies reflect very early cancers or, alternatively, merely point to a cancer risk rather than the presence of disease. The smoking status of our normal subjects and patients with cancer was unknown. However, the p53 responses in this study were low and removal of this antigen from the panel was not found to significantly change the sensitivity or specificity of the panel for lung cancer. Autoantibodies to cancer-associated antigens other than p53, in people who smoke have not been reported.

Raised levels of autoantibodies, and particularly multiply raised autoantibodies were rarely seen in these normal plasma samples, and this low level of tumour-associated autoantibodies is in agreement with other publications. ${ }^{72} 22$ No further information was available for the control group, including smoking history, so the possibility that one or more of these controls might have been harbouring an occult malignancy cannot be ruled out.

The potential clinical significance of autoantibodies for screening and early diagnosis appears to lie in combining an optimised panel of assays to measure these autoantibodies. The advantage of this approach is that it should be possible to detect the full range of heterogeneous lung cancers by increasing the number of autoantibody assays or altering the antigens used in the autoantibody panel, and with the evidence for autoantibodies present to antigens such as livin, survivin and annexins I and II, p62 and Hsp40, ${ }^{74-37}$ as well as an increasing number of other cancer-specific immunoreactive antigens being described every year, a potential diagnostic assay using measurement of autoantibodies in subjects "at risk" should be available within a short period of time.

A diagnostic test for lung cancer is of particular importance owing to the late stage at which patients currently present with this disease and the fact that this disease will cause significant social burden for at least 20 years, even if all smoking were discontinued today. Obviously, the potentially harmful effects of any test must be taken into account when deciding on its suitability. The lungs are very sensitive to radiation so frequent $x$ ray examinations are not an ideal screening test. For screening to be introduced, a test needs to be simple, quick, inexpensive and beneficial so that it can be used on people at high risk of cancer development. A blood test, such as that described here, is non-invasive, cost effective relative to imaging tests, carries no side effects and is acceptable to the vast majority of patients.

The levels of sensitivity and specificity demonstrated here suggest that the measurement of autoantibodies could act as an aid to cancer diagnosis. Such a test could be performed on the peripheral blood of people with an increased risk of developing cancer as a means of selecting patients for an imaging test. People with a positive autoantibody test would proceed to spiral $\mathrm{CT} /$ magnetic resonance imaging, which if it confirmed a lung (or other) cancer would then lead to treatment. Imaging negative patients would continue to be intensively followed up in specialist clinics.

Further studies are planned using an increased assay panel which will include some TAAs thought to be more specific for lung cancer. These will be tested on samples taken from earlier stage cancers as well as prediagnostic samples using agematched controls with known smoking histories.

Acknowledgements: We thank Natalie Colborne for technical assistance, and Susan McKendrick, CStat, for statistical advice.

Funding: This work was supported in part by funding from the 5th Framework Programme of the EU (EUCIP), the University of Nottingham and Oncimmune Ltd.

Competing interests: None declared. 


\section{REFERENCES}

1. Office for National Statistics. Twentieth century mortality - 95 years of mortality data in England and Wales by age, sex, year and underlying cause. London: Office for National Statistics, 1997 (ISBN 1857742 397.)

2. Felip $\mathbf{E}$, Pavlidis N, Stahel RA, cooordinating authors for the ESMO guidelines task force. ESMO Minimal Clinical Recommendations for diagnosis, treatment and followup of small-cell lung cancer (SCLC). Ann Oncol 2005;16(suppl 1):i30-1.

3. Felip E, Stahel RA, Pavlidis N, cooordinating authors for the ESMO guidelines task force. ESMO Minimal Clinical Recommendations for diagnosis, treatment and followup of non-small-cell lung cancer (NSCLC). Anns Oncol 2005;16(suppl 1):i28-9.

4. MacRedmond R, McVey G, Lee M, et al. Screening for lung cancer using low dose CT scanning: results of 2 year follow up. Thorax 2006;61:54-6.

5. Ries LAG, Eisner MP, Kosary CL, et al SEER cancer statistics review, 1975-2001. Bethesda, MD: National Cancer Institute.

6. Zhong L, Coe SP, Stromberg AJ, et al. Profiling tumor-associated antibodies for early detection of non-small cell lung cancer. J Thor Oncol 2006;1:513-9.

7. Zhang JY, Casiano CA, Peng XX, et al. Enhancement of antibody detection in cancer using panel of recombinant tumor-associated antigens. Cancer Epidemiol Biomarkers Prev 2003;12:136-43.

8. Storr SJ, Chakrabarti J, Barnes A, et al. Use of autoantibodies in breast cancer screening and diagnosis. Expert Rev Anticancer Ther 2006;6:1215-23.

9. Robertson JFR, Chapman C, Cheung K-L, et al. Autoantibodies in early breast cancer. J Clin Oncol 2005;23(Suppl 1):549.

10. Robertson JFR, Graves RL, Price MR, Inventors; Onclmmune Ltd, assignee. Tumour markers. European patent EP-B 1078264, 2005.

11. Li Y, Karjalainen A, Koskinen $\mathrm{H}$, et al. p53 Autoantibodies predict subsequent development of cancer. Int J Cancer 2005;114:157-60.

12. Chapman C, Murray A, Chakrabarti J, et al. Autoantibodies in breast cancer: their use as an aid to early diagnosis. Ann Oncol 2007:18:868-73.

13. Crawford LV, Pim DC, Bulbrook RD. The detection of antibodies against the cellular protein p53 in sera from patients with breast-cancer. Int J Cancer 1982;30:403-8.

14. Sangrajrang S, Sornprom A, Chernrungroj G, et al. Serum p53 antibodies in patients with lung cancer: correlation with clinicopathologic features and smoking. Lung Cancer 2003;39:297-301.

15. Li Y, Brandt-Rauf PW, Carney WP, et al. Circulating anti- p53 antibodies in lung cancer and relationship to histology and smoking. Biomarkers 1999;4:381-90.

16. Trivers GE, DeBenedetti VM, Cawley HL, et al. Anti p53 antibodies in sera from patients with chronic obstructive pulmonary disease can predate a diagnosis of cancer. Clin Cancer Res 1996:2:1767-75.

17. Veale D, Ashcroft T, Marsh C, et al. Epidermal growth factor receptors in non-small cell lung cancer. Br J Cancer 1987;55:513-6.

18. Szelachowska J, Jelen M, Kornafel J. Prognostic significance of intracellular laminin and Her2/neu overexpression in non-small cell lung cancer. Anticancer Res 2006;26:3871-6.

19. Awaya H, Takeshima Y, Yamasaki M, et al. Expression of MUC1, MUC2, MUC5AC, and MUC6 in atypical adenomatous hyperplasia, bronchioloalveolar carcinoma, adenocarcinoma with mixed subtypes, and mucinous bronchioloalveolar carcinoma of the lung. Am J Clin Pathol 2004;121:644-53.

20. Hirasawa Y, Kohno N, Yokoyama A, et al. Natural autoantibody to MUC1 is a prognostic indicator for non-small cell lung cancer. Am J Respir Crit Care Med 2000;161:589-94.

21. Krause $\mathbf{P}$, Tureci 0 , Micke $P$, et al. SeroGRID: an improved method for the rapid selection of antigens with disease related immunogenicity. J Immunol Methods 2003;283:261-7.

22. Türeci $\mathbf{0}$, Mack $U$, Luxemberger $U$, et al. Humoral responses of lung cancer patients against tumor antigen NY-ESO-1. Cancer Lett 2006;236:64-71.

23. Cho B, Lim Y, Lee DY, et al. Identification and characterization of a novel cancer/ testis antigen gene CAGE. Biochem Biophys Res Commun 2002;292:715-26.

24. Cho B, Lee H, Jeong $S$, et al. Promotor hypomethylation of a novel cancer/testis antigen gene CAGE is correlated with its abberant expression and is seen in premalignant stage of gastric cancer. Biochem Biophys Res Commun 2003;307:5263.

25. Xia 0, Kong XT, Zhang GA, et al. Proteomics-based identification of DEAD-box protein 48 as a novel autoantigen, a prospective serum marker for pancreatic cancer. Biochem Biophys Res Commun 2005;330:526-32.

26. Tureci 0, Usener D, Schneider S, et al. Identification of tumor-associated autoantigens with SEREX. Methods Mol Med 2005;109:137-54.

27. Linder P. Dead-box proteins: a family affair - active and passive players in RNPremodeling. Nucleic Acids Res 2006;34:4168-80.

28. Sambrook J, Fritsch EF, Maniatis T. Molecular cloning a laboratory manual. ed 2. USA: Cold Spring Harbour Laboratory Press, 1989.

29. Gendler SJ, Spicer AP, Lalani EN, et al. Structure and biology of the carcinomaassociated mucin, MUC1. Am Rev Resp Dis 1991;144:S42-7.

30. Armitage $\mathbf{P}$, Berry G, Matthews JNS. Statistical methods in medical research. 4th ed. Oxford, UK: Blackwell Science, 2002: 117.

31. Nesterova M, Johnson N, Cheadle C, et al. Autoantibody biomarker opens a new gateway for cancer diagnosis. Biochim Biophys Acta 2006;1762:398-403.

32. Neri M, Betta $P$, Marroni $P$, et al. Serum anti-p53 autoantibodies in pleural malignant mesothelioma, lung cancer and non-neoplastic lung diseases. Lung Cancer 2003:39:165-72

33. Gure A0, Chua R, Williamson B, et al. Cancer-testis genes are coordinately expressed and are markers of poor outcome in non-small cell lung cancer. Clin Cancer Res 2005:11:8055-62.

34. Megliorino R, Shi FD, Peng XX, et al. Autoimmune response to anti-apoptotic protein survivin and its association with antibodies to p53 and c-myc in cancer detection. Cancer Detect Prev 2005:29:241-8.

35. Brichory $\mathbf{F M}$, Misek DE, Yim AM, et al. An immune response manifested by the common occurrence of annexins I and II autoantibodies and high circulating levels of IL-6 in lung cancer. Proc Natl Acad Sci USA 2001;98:9824-9.

36. Yagihashi A, Asanuma K, Kobayashi D, et al. Detection of autoantibodies to livin and survivin in sera from lung cancer patients. Lung Cancer 2005:48:217-21.

37. Oka M, Sato S, Soda H, et al. Autoantibody to heat shock protein Hsp40 in sera of lung cancer patients. Jpn J Cancer Res 2001;92:316-20. 
Therapeutic CPAP improved glycaemic control after 3 months in our subjects with diabetes. Changes in body composition may play a role. Unfortunately, bioelectrical impedance analysis, as used in all studies, has its limitations. ${ }^{3}$

It would be very interesting to know whether there is an effect of CPAP therapy on insulin sensitivity in less obese diabetic subjects as we demonstrated a rapid improvement in insulin sensitivity in our study in the non-diabetic OSAS group in those with a BMI $<30 \mathrm{~kg} / \mathrm{m}^{2}$. That this early effect of CPAP may be related to acclimatisation to the conditions of the sleep laboratory and the clamp procedure is questionable as our studies were done under exactly the same conditions and there is no reason to postulate a higher stress sensitivity in leaner patients.

Although we could not measure plasma catecholamines, we were able to re-measure serum cortisol as another marker of sympathetic stimulation in 20 individuals in our study, ${ }^{1}$ and could not find significant differences before (mean 19.18 (SD 3.52) $\mu \mathrm{g} / \mathrm{dl}$ ) and 2 days after $(19.35(3.27) \mu \mathrm{g} / \mathrm{dl})$ onset of CPAP therapy $(p=0.59)$.

\section{A Harsch, E G Hahn, S Pour Schahin}

Medical Department 1, Friedrich-Alexander University of Erlangen-Nuremberg, Erlangen, Germany

Correspondence to: Dr I A Harsch, Department of Medicine I, Friedrich-Alexander University ErlangenNuremberg, Ulmenweg 18, 91054 Erlangen, Germany; igor.harsch@uk-erlangen.de

Competing interests: None.

Thorax 2008;63:384-385. doi:10.1136/thx.2007.090415

\section{REFERENCES}

1. Harsch IA, Pour Schahin S, Radespiel-Tröger M, et al. Continuous positive airway pressure treatment rapidly improves insulin sensitivity in patients with obstructive sleep apnea syndrome. Am J Respir Crit Care Med 2004;169:156-62.

2. Harsch IA, Schahin SP, Brückner K, et al. The effect of continuous positive airway pressure treatment on insulin sensitivity in patients with obstructive sleep apnoea syndrome and type 2 diabetes. Respiration 2004;71:252-9.

3. Bioelectrical impedance analysis in body composition measurement. NIH Technol Assess Statement Online 1994 December 12-14. http://www.ncbi.nlm.nih.gov/ books/bv.fcgi?rid = hstat4. section.26000 (accessed 15 January 2008).

\section{Authors' reply}

We thank Harsch et al for their comments. Their letter highlights the important contribution of obesity in studies of both insulin resistance and obstructive sleep apnoea (OSA). Although obesity underlies both pathologies, it also confounds studies investigating these conditions. The only studies therefore that can determine conclusively the effect of continuous positive airway pressure (CPAP) on improvements in insulin resistance and glycaemia in patients with OSA are double blind randomised controlled trials. We agree that a randomised controlled trial of CPAP in less obese subjects with type 2 diabetes would clarify this area further, but a study of pre-diabetic subjects with insulin resistance would be even more enlightening.

\section{S West, J Stradling}

Oxford Centre for Respiratory Medicine, Churchill Hospital, Oxford, UK

Correspondence to: Professor J Stradling, Oxford Centre for Respiratory Medicine, Churchill Hospital, Oxford OX3 7LJ, UK; john.stradling@orh.nhs.uk

Competing interests: None.

\section{Mould eradication and asthma}

The paper by Burr et $a l^{1}$ on the efficacy of eradicating visible indoor mould on respiratory health in patients with asthma is of great interest, but I think the authors underestimate the clinical relevance of their findings because they overestimate the lack of effect on peak expiratory flow (PEF) variability as an objective assessment of their intervention. The lack of effect on this primary end point in the presence of highly significant effects on medication use and symptoms - even after 12 months-simply illustrates once again that PEF is too insensitive to contribute meaningfully to the interpretation of our therapeutic interventions. The study by Burr et $a l^{1}$ and those of others ${ }^{23}$ are examples of investigations that demonstrate a lack of efficacy using PEF parameters as primary end points whereas the secondary end points-such as respiratory symptoms-demonstrate efficacy of the interventions. Increased PEF variability is a specific feature of unstable asthma but it is not necessarily a sensitive one. PEF mainly reflects central airway mechanics ${ }^{4}$ and is therefore not the optimal monitoring tool because asthma predominantly affects the smaller airways. Hence, PEF may severely underestimate peripheral airway patency. Clinical studies are much more convincing and powerful if sensitive and relevant end points are chosen, and I would strongly advocate using end points that are both relevant and sensitive. This will teach us more and provide more credit for all involved-doctors as well as patients.

\section{P J F M Merkus}

Correspondence to: Dr P J F M Merkus, Radboud University Children's Hospital, P 0 Box 9101, Nijmegen 6500 HB, The Netherlands; p.merkus@cukz.umcn.nl

Competing interests: None.

\section{REFERENCES}

1. Burr ML, Matthews IP, Arthur RA, et al. Effects on patients with asthma of eradicating visible indoor mould: a randomised controlled trial. Thorax 2007;62:767-72.

2. Papi A, Canonica GW, Maestrelli P, et al. Rescue use of beclomethasone and albuterol in a single inhaler for mild asthma. N Engl J Med 2007;356:2040-52.

3. Boushey HA, Sorkness CA, King TS, et al. Daily versus as-needed corticosteroids for mild persistent asthma. N Engl J Med 2005;352:1519-28.

4. Pedersen OF, Brackel HJ, Bogaard JM, Kerrebijn KF. Wave-speed-determined flow limitation at peak flow in normal and asthmatic subjects. J App/ Physiol 1997;83:1721-32.

\section{CORRECTIONS}

doi:10.1136/thx.2006.077081 corr1

The label "OR (odds ratio)" was erroneously introduced into the headings to tables 3 and 4 in the paper by Aldington et al (Thorax 2007;62:1058-63). In table 3, the numbers in the columns refer to the estimates of the difference of the particular measurement of respiratory function between those who do and those who do not smoke tobacco, and those who do and do not smoke cannabis, respectively. The heading in table 4 refers incorrectly to OR for association between tobacco pack years or cannabis joint years and the measurement of respiratory function. The numbers in the columns refer to the change in the particular measurement of respiratory function per unit change of pack years and joint years respectively. The "OR" label should be omitted from these tables.

\section{doi:10.1136/thx.2007.083592corr1}

We would like to draw readers' attention to a typographical error in the article by Chapman et al (Thorax 2008;63:228-33). In the discussion, the antigen CAGE is referred to as CAGE (DDX58) and should read CAGE (DDX48); however, the corresponding references are correct. The section is given in full below:

"The DEAD-box cancer testis antigen CAGE (DDX48) has previously been shown to be expressed in a number of cancers including gastric, cervical and lung cancer tissue and cell lines, and autoantibodies have been reported to this protein in some but not all of the cancers samples studied. ${ }^{25 "}$ 